\title{
Community Tools for Repurposing Learning Objects
}

\author{
Chu Wang ${ }^{2}$, Kate Dickens ${ }^{1}$, Hugh C Davis ${ }^{2}$, and Gary Wills ${ }^{2}$ \\ ${ }^{1}$ eLanguages, University of Southampton, UK \\ kate.dickens@soton.ac.uk \\ ${ }^{2}$ School of Electronics and Computer Science, University of Southampton, UK \\ $\{$ cw2, hcd, gbw $\}$ @ecs. soton. ac.uk
}

\begin{abstract}
A critical success factor for the reuse of learning objects is the ease by which they may be repurposed in order to enable reusability in a different teaching context from which they were originally designed. The current generation of tools for creating, storing, describing and locating learning objects are best suited for users with technical expertise. Such tools are an obstacle to teachers who might wish to perform alterations to learning objects in order to make them suitable for their context. In this paper we describe a simple set of tools to enable practitioners to adapt the content of existing learning objects and to store and modify metadata describing the intended teaching context of these learning objects. We are deploying and evaluating these tools within the UK language teaching community.
\end{abstract}

Keywords: Learning objects, Community of Practice, repurposing, Wiki, contextual metadata.

\section{Introduction}

Widespread web-based learning has drawn educators' attentions to the concept of learning objects. Polsani [1] has defined a learning object as "an independent and self-standing unit of learning content that is predisposed to reuse in multiple instructional contexts". This definition suggests several functional requirements which are essential for creating sensible learning objects. For example [1]:

- Accessibility (tagging with metadata so that it can be stored and referenced in a database);

- Reusability (functioning in different instructional contexts);

- Interoperability (should be independent of both the delivery media and knowledge management systems).

Of particular importance is sharing and reusing e-learning materials, which may lead to an improvement in the quality of teaching; the sharing of good practice; greater consistency and an enhanced sense of community [2]. Despite the various forms a learning object might take, digital or non-digital, in the scope of this paper we aim at tackling learning objects with HTML format. The community that we work with consists of language teachers and instructors who engage in producing pedagogically sound online learning objects but have limited technical expertise. In 
this community, reusing online learning materials in different instructional contexts is a very common practice: it is traditional practitioners' experience in adapting teaching materials to suit different student groups.

When learning objects are reused in different educational scenarios, their context related information often changes. These contexts represent information about the intended target audience, the purpose of the teaching, instructional methods, pedagogic approach being used and so on. For example, teachers might take an existing learning object targeted at teaching nursing students study skills, to produce a different version of the same material targeted at engineering students; or teachers can constrain the size of certain materials to be viewed on different devices such as PDAs or mobile phones. With the change of context, learning objects have to be repurposed, generally, in terms of both their content and the metadata describing the nature of the learning objects. Metadata facilitates the identification, search and retrieval of learning objects and can be extended to represent different instructional contexts. IMS/GLC ${ }^{1}$ has provided specifications and guidelines for metadata standards and extension rules. However, standard metadata is not sufficient to identify particular learning objects in our domain. Hence, extra context-rich metadata [3], in other words contextual metadata, plays an important role in promoting the reusability of a learning resource. Here context has been defined as "A set of circumstances in which a learning object is used or may be used" [4]. Particularly the context refers to the teaching and learning circumstances. Learning objects with such information attached are able to be identified by their context and then repurposed to suit different needs.

Inevitably challenges exist while reusing learning objects in differing contexts [5] especially in relation to issues about extensions on relevant standards, exchange formats for the contextualization of resources, and the creation of tools for development of contextualized learning resources. In particular, our community practitioners indicate that they find it problematic to repurpose learning objects in terms of both content and metadata. Current available tools such as Macromedia Dreamweaver ${ }^{\mathrm{TM}}$ could be used to edit learning object content; unfortunately, nontechnical practitioners find this software complex and therefore difficult to use without proper training. Besides it is only available to licensed users and this means that not every practitioner has access to it. An open source tool, RELOAD ${ }^{2}$, which was designed for editing learning object metadata and content packaging, has been used by community members. However, it is not particularly user-friendly, especially for users with limited technical capabilities. Such users often find it frustrating to use. Therefore a need has been identified for an easy-to-use and accessible tool. It is the authors' intention to address such a need by designing a novel toolkit to use with an existing repository of language learning objects in HTML format, and to test with an established Community of Practice.

The remainder of this paper is organized as follows: In section 2, we review issues of the contextual metadata and related work. Section 3 outlines the proposed toolkit with which users can repurpose learning content and metadata and discover relevant objects. In section 4 we illustrate how we developed an innovative Wiki-type editor for content repurposing; while section 5 and section 6 describe a metadata facilitator

\footnotetext{
${ }^{1}$ http://www.imsglobal.org/

${ }^{2}$ http://www.reload.ac.uk
} 
in aiding users to modify learning object metadata easily, and a tool to help in the discovery of relevant learning objects to be repurposed. Section 7 reports the feedback from an initial evaluation. Finally the authors conclude the paper and explain the ongoing work in the project.

\section{Contextual Metadata and Related Works}

The last few years have seen a debate arise between the approaches to designing learning objects in contextualized and "de-contextualized" scenarios [4, 6, 7]. Some resources need to be designed to be context-neutral in order that learners from any subject area can easily engage with them. On the other hand, the contextualization of a learning object is beneficial to the learners, as the learning object can then be seen as more relevant to the subject being studied [6]. Learning objects are widely developed as free from the context of teaching and learning to facilitate interoperability, despite evidence that shows this to be contrary to teachers' needs (as from our community experience). It is our view that context is becoming increasingly important with the advent of IMS Learning Design and the use of other ontologies. We decided to extend the standard metadata with a set of contextualized metadata since the constrained taxonomy from international standards is not sufficient or accurate enough to describe our instructional context. Brooks and McCalla [8] has proposed an inspiring ecological approach to capture a larger set of end-use information as metadata in order to overcome the rigidities of standard approaches and assist software agents carry out automatic processing. Their work helped us form a better view of defining and collecting valuable contextual metadata, however we would still adopt standards by mapping our contextual metadata to $\mathrm{LOM}^{3}$ to retain the interoperability of the learning objects amongst repositories.

Research carried out within the eLanguages group at Southampton ${ }^{4}$ found that contextual metadata facilitates greater scope for reuse, and this was verified by tests conducted with our teacher/instructor communities. Indeed we are not alone, as several other projects have investigated the issues related to the application of contextualized metadata. For example, the RAFT project discovered that for mobile learning to be effective, standards and exchange formats need to be extended to include contextual data [5]. Providing subject specific information is another valuable usage for contextual metadata. One way to add context to learning objects, without limiting their usability and reusability, is by assigning metadata relevant to specific communities of practice. We discovered that it helps teachers to identify learning objects more effectively by attaching language specific metadata so that they can find the right learning material to reuse. Similar work has been carried out by the DocSouth digital library in considering the biographical and geographic metadata requirements of teachers [9]. Recent work has been undertaken to produce a repository query language, ProLearn Query Language (PLQL) ${ }^{5}$. The addition of a keyword-based search mechanism to extract learning objects that best match informal descriptions, would suit repositories that enable rich annotation of learning objects.

\footnotetext{
${ }^{3}$ http://ltsc.ieee.org/wg12/files/LOM_1484_12_1_v1_Final_Draft.pdf

${ }^{4} \mathrm{http}: / /$ www.elanguages.ac.uk/research

${ }^{5}$ http://ariadne.cs.kuleuven.be/lomi/index.php/QueryLanguages
} 
Such repositories can treat contextual metadata as informal descriptions, and including this information when preforming searches would enhance resource discovery. Our work draws on the experiences gained in these projects.

In the eLanguages $\mathrm{L}_{2} \mathrm{O}$ project [3], Reusable Learning Objects (RLOs) contain extensions to UK LOM CORE ${ }^{6}$ Metadata standards that directly address the needs of the Modern Languages Teaching Community of Practice. The learning objects are assembled in a content package with their associated pedagogic and technical assets and relevant metadata. The contextual metadata includes points of pedagogic information to guide a user, such as the "description for learners", "suggestions for further use" or "language specifics". Reuse is greatly enabled through this additional metadata, which describes the pedagogic nature of the learning objects.

\section{Toolkit Overview}

The MURLLO (Management, Use and Repurposing of Language Learning Objects) [10] project aims to tackle concerns surrounding the effective repurposing of RLOs. We have proposed a system with three components/tools to deal with these concerns in our community (as shown in Fig. 1):

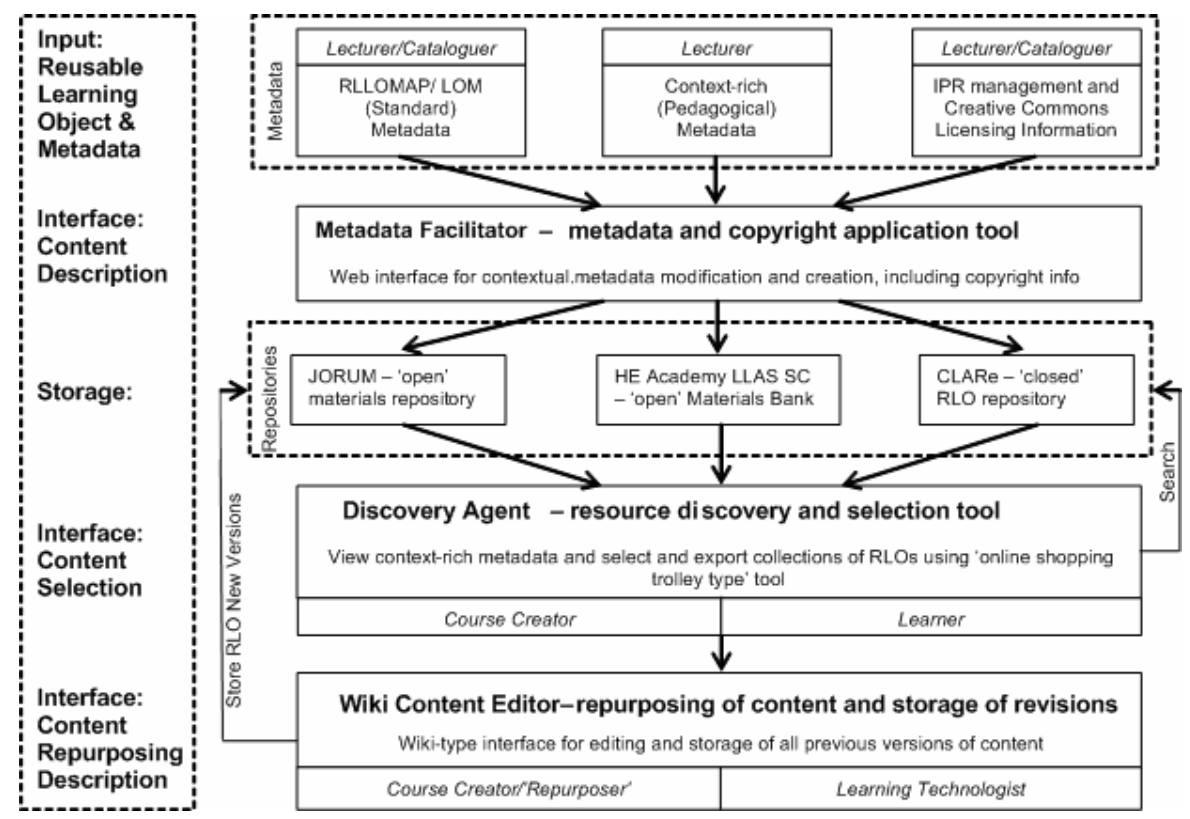

Fig. 1. The MURLLO System Architecture

- The Wiki-Type Content Editor allows repurposing of content, without the need for extra editing tools, and the storage of this content and its revisions.

\footnotetext{
${ }^{6}$ http://zope.cetis.ac.uk/profiles/uklomcore
} 
- The Metadata Facilitator acts as an interface to allow a teacher-friendly application of customized context-rich metadata to RLOs and facilitates storage of resultant content packages in open and closed repositories.

- The Discovery Agent facilitates resource discovery by displaying contextual metadata and by allowing selection and export of collections of RLOs.

Learning objects produced in the community of language teachers with whom we are working are written in HTML, which appear as web pages. The Wiki style editor is a critical component which enables a non-technical user to adapt this type of learning objects. It directly affects how well users can achieve their repurposing targets. We will focus on the Wiki-type tool in this paper.

\section{The Wiki-Type Content Editor}

As pointed out in [11], the ease of collaboration in a Wiki can make it a powerful tool for project management and collaborative writing. In our case, the Wiki-type editing tool would provide the necessary facility to adapt and repurpose learning objects. First of all, the motivation for such a tool has been identified and is explained here.

\subsection{The Need for Wiki Editing}

There is research $[6,12]$ which focuses on design patterns or best practice for learning objects intended for reuse in other contexts. However, we have a different perspective in terms of reuse. The previous $\mathrm{L}_{2} \mathrm{O}$ project has shown that it is easy to reuse pedagogic assets such as stand-alone audio files. However, it might be difficult for a non-technical educator to adapt HTML content within online learning objects for different target audiences. The need for an easy-to-use editing tool has been stressed many times by our community of users.

Our community comprises practitioners from four regional universities in UK Higher Education (HE) sector. They are mostly language teachers but also some learning technologists and researchers. Most of the practitioners did not have any previous experience of creating learning objects, and even though they have started to share and adapt learning materials stored in the community repository in $\mathrm{L}_{2} \mathrm{O}$, they still need technical support because of their limited knowledge of web related technologies. Therefore, demands for a tool to support the adaptation of online learning objects according to instructional contexts have emerged. This fits in with traditional practitioners' experience as previously mentioned (this finding was also supported by the attendees of an extended workshop ${ }^{7}$ ).

Furthermore, the concept of collaborative development of learning objects in the sense that different types of experts may need to cooperate to author or adapt an object has been suggested [1]. These experts can be programmers, graphic designers and subject experts/teachers. Similarly we discovered that, in a collaborative online environment, expertise can be shared, exchanged and viewed by the community in order to achieve adapting and editing goals. With these views in mind, a Wiki, a type

\footnotetext{
${ }^{7}$ e-Learning conference, http://www.llas.ac.uk/events/llaseventarchiveitem.aspx?resourceid= 2654\#day2
} 
of Web server that allows any reader of its pages to edit those pages, or create new ones using simple web forms [13], can provide an ideal collaborative editing environment. Meanwhile keeping different versions of adapted learning objects can help the community to track and view changes if needed. To rectify the current lack of relevant tools, the MURLLO project has proposed a Wiki-type editor to fill the gap. The requirements from the user community for such an editor are outlined in the next section.

\subsection{The Wiki-Type Editor Requirements}

A learning object produced within our Community of Practice usually comprises one or more HTML files defining the content; media files to be referred to by the HTML files; a set of metadata in XML format which needs to be attached to the learning object in order to comply with relevant learning object standards (IMS, IEEE LOM), together with corresponding schema definition files. All of these content resources are assembled in one package. Considering the structure of this type of package and the technical compentency of our user community, a Wiki-type editor specifically for adapting learning objects would require the following features:

- A WYSIWYG content editing environment suitable for non-technical users, as it is easy to use and has powerful formatting capabilities so that users do not need to worry about the underlying HTML syntax;

- A versioning system that creates a new version after a learning object is modified and stores all previous versions of learning objects;

- Viewing and rollback of earlier versions so that a version can be reverted if necessary;

- An edit summary showing the nature of the changes, to be used as a metadata field defining the relationships between different versions of the same learning object;

- The ability to handle embedded media objects and allow relatively easy editing of these components;

- An importing facility which allows users to upload learning objects (preferably as a content package) from local computers or external sources; and an exporting function by which users can select and download repurposed learning objects to local computers or external sources; these facilities will be linked with the metadata facilitator and discovery agent at a later stage.

\subsection{The Design Choice for the Wiki-Type Editor}

According to the requirements listed above, it was a natural inclination for our team to consider using or adapting an existing Wiki system as the learning object editor. In order to choose the most appropriate solution, we looked into a number of different Wiki environments, including Mediawiki, DokuWiki, Zwiki and many more; a Document Management System (DMS), TWiki; and Content Management Systems (CMSs), Silva and Plone. We compared some of the important features including user friendly WYSIWYG toolbars, versioning capability, and their ability to handle embedded media. 
Although quite a few of them seemed to partially match with the above criteria, we were confronted with difficulties when importing the existing learning objects into the Wiki systems. The learning object content are basically web pages which are composed of standard HTML markup (with links to images, multimedia and embedded Shockwave Flash ${ }^{\mathrm{TM}}$ objects), whereas Wikis are created using their own markup language which is totally different from HTML. Therefore a learning object always needs to be converted into the format used by the Wiki before it can be used in the Wiki editor. It would also still need to be converted back to HTML before the learning object could be exported from the system. The underlying problem was that the "round-trip" conversion between HTML and Wiki markups causes a loss of information from the HTML web pages, especially embedded object tags and snippets defining page styles. For DMS/CMS (with built-in HTML editors), it was not necessary to convert between Wiki and HTML but the style information or embedded object tags did not display properly. If this information is not retained, both look-andfeel and functionality is affected. This would have a serious impact on users since they would be unable to repurpose materials if they could not see how the original learning objects had appeared. Furthermore, there were some other critical problems such as the lack of package importing functions for most Wikis and the unsuitable versioning facilities for the CMS systems.

As none of the above tools particularly fitted our requirements, we came to the conclusion that it would be more efficient to design our own system by integrating an HTML editor and to develop other essential functions like versioning and import/export facilities around it. The implementation of this solution is presented in the following section.

\subsection{The Wiki-Type Editor Implementation and Usage}

We decided to use a lightweight but powerful HTML editor, FCKeditor ${ }^{8}$, to integrate a Wiki-type online editing workspace for our community. We labelled it "Wiki-type", since we have replicated a Wiki's collaborative authoring characteristics around a regular HTML editor. It is intended for peer-editing of existing learning objects but is not a "Wiki" in the more general sense of the term. As it was designed for teachers who have no web expertise, we have made the interface as intuitive as possible. The choice of HTML editor made the implementation process relatively smooth and with certain customisations it was turned into an effective editing tool for learning objects. A learning object can be uploaded as a standard IMS content package, and then the system handles de-packaging which separates the learning object content from metadata and other documents. The only step users need to take is to locate the learning object from an index page and click the title link which will lead to the learning object being displayed in their browser. The look-and-feel of a learning object is kept consistent even in the editing mode so that novice users can edit it without being confused by the underlying HTML syntax and can also edit the content as it appears to their students.

There are three possible types/levels of modifications a typical user might make to a learning object:

\footnotetext{
${ }^{8}$ http://www.fckeditor.net/
} 
- Minor corrections which would not generally affect/change the nature of learning objects, including spelling, grammar, re-organization of sentences and so on. In this case, the metadata does not usually require amendments.

- More substantial modifications in terms of pedagogical design, which result in the possible re-ordering of content, alteration of activities or wording. In this case the relevant metadata would probably need to be modified accordingly.

- Complete repurposing of a learning object to be used in a very different instructional context, with dramatic changes in content, order, or style. For example, a new object might be made based on the template of an existing learning object but the theme and content diverge from the original. In this case, the learning object undergoes major changes and some or all of the metadata must be modified.

According to the above types of modifications, one issue in the development process was to decide whether to make new versions for each minor/major modification, or only associate each major change (changes affecting pedagogical design) with a new version. After careful consideration, the decision was made that versions should always be kept rather than overwriting existing copies no matter how many changes had been made. As some modifications to original learning objects could be controversial or subjective and may only reflect personal opinions, it is wise to keep a full version history with rollback facility, so that a faulty change can be reversed. Another decision we made was to consider versioning learning objects as whole packages, containing web pages, media attachments and metadata, not as in traditional Wikis, where versions are handled at page level (single document). Hence package level versioning was adopted to accommodate the nature of learning objects and to allow future extension on the toolkit to edit not only content but also metadata and other attachments in content packages. In this way, the learning objects are always treated as self-contained units of standard content packages. A user imports learning objects as stand-alone packages and similarly a package is generated automatically when a user creates a new version or exports an object. The packaging and disassembling are carried out behind the scenes so non-technical users do not need to get involved in the process.

The figures below show some scenarios using the Wiki-type editor. Fig. 2 and Fig. 3 show the same learning object in viewing mode (original look) and in editing mode. They look almost identical apart from the user-activated feedback mechanism (Show answer/Hide answer link in Fig. 2 and 3 below), which is expanded in the editing mode so that a user can easily change the text in the answer panel. A user can also make more complicated changes using the formatting toolbar near the top of the browser: from editing an image, adding a new multiple choice question, replacing an existing linked document with an updated version, to adding a new learning activity.

Scenario 1, illustrated by the figures below, presents that a learning object in the English language (Fig. 4) has been repurposed to be used by German students (see Fig. 5). Therefore they have the exact same content and subject but are represented in different languages in order to target different student groups. It can be easily adapted in the Wiki-type editor by simply replacing the English text with the equivalent German. Fig. 5 also shows how the text in the "Select your answer" box can be replaced straightforwardly by using the selection field toolbar. 


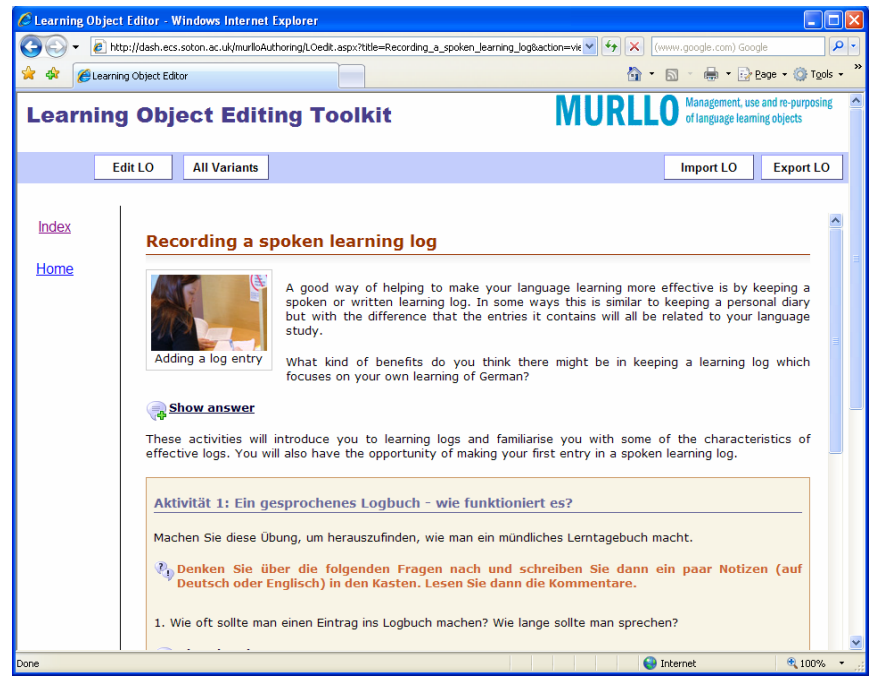

Fig. 2. A learning object in viewing mode

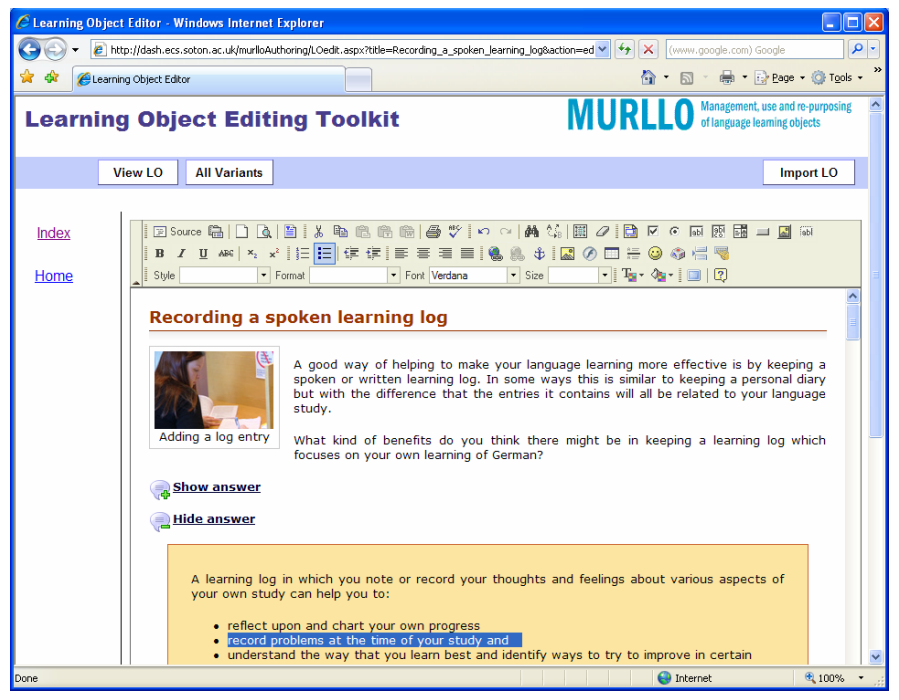

Fig. 3. A learning object in editing mode 


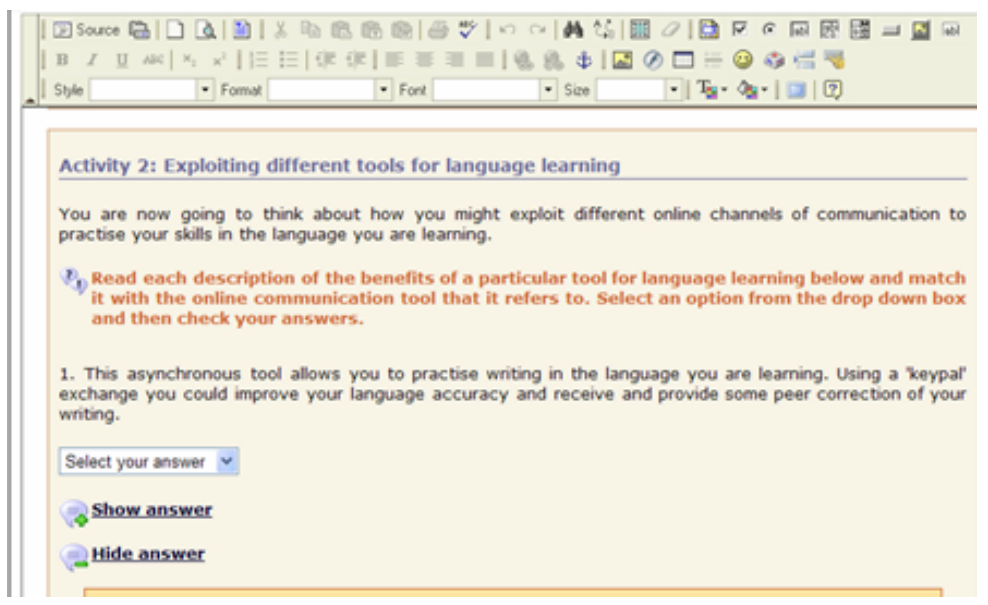

Fig. 4. A snapshot of learning object (English version)

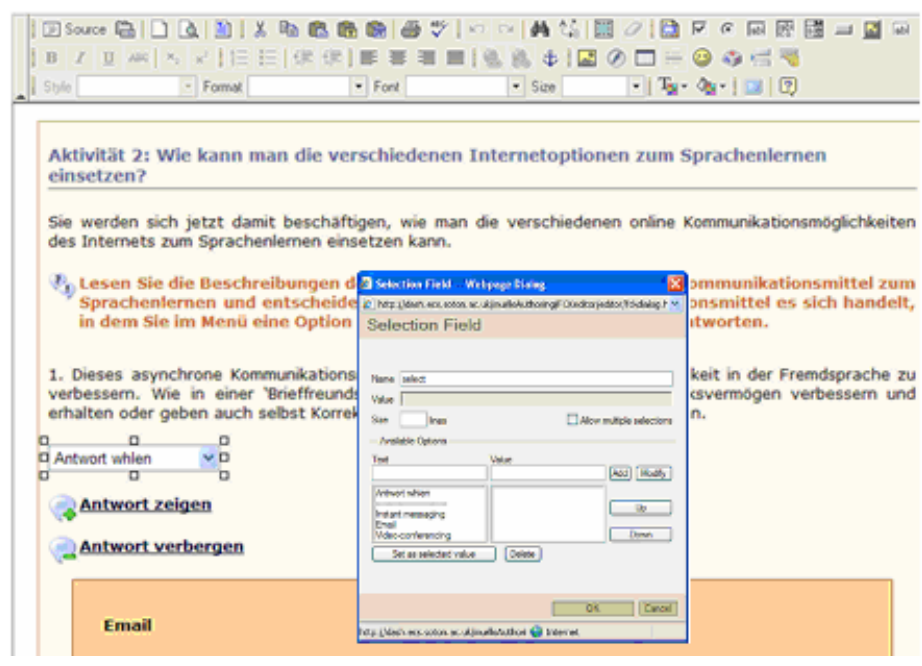

Fig. 5. A snapshot of learning object (Equivalent German version)

\section{Metadata Facilitator}

Repurposing learning objects not only requires content editing but also necessitates relevant modifications of metadata or contextual metadata in many cases. In the previous section, an example of repurposing a learning object was to switch the presentational language of the learning object from English to German (scenario 1). There can be many different ways of adapting learning objects using the editing tool. Here are some other possibilities: 
- Scenario 2: practitioners can adapt aspects of learning objects to suit a particular purpose or learning environment. For instance, to personalize materials (e.g. images) for use in a particular course or institution, or to reflect different departmental policies on referencing and citation.

- Scenario 3: practitioners can add a new activity including, for example, a set of assessment questions following the current template and style.

Our experience indicates that both adaptations in scenario 1 and 2 would require relevant modifications of metadata or contextual metadata. For instance, in scenario 1 the metadata related to description, language and targeted audience need to be changed (Fig. 6 is an excerpt of the metadata and Table 1. shows the changes in metadata); and the description for learners or the author information would probably need to be altered in scenario 2 . In scenario 3 , it is not necessary to amend the metadata record if this modification is just an update of assessment questions in the existing learning object.

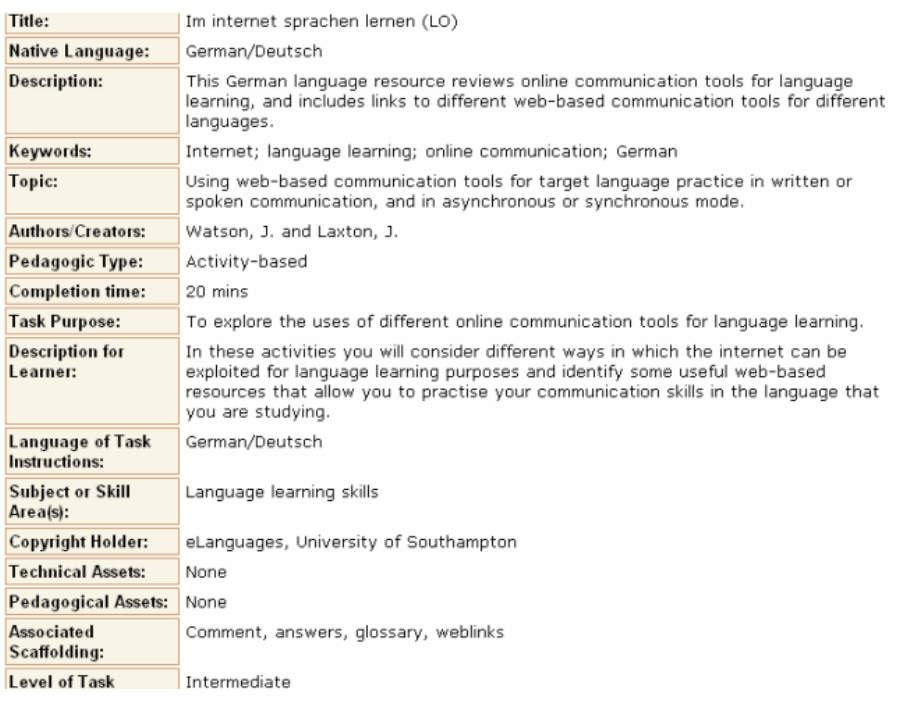

Fig. 6. An excerpt of the metadata for the German version

According to our community experience, adding or changing metadata can be extremely difficult for non-technical users without the support of proper tools. The best available open source tool, RELOAD, is not particularly easy to use as the language teachers in our community found; moreover the metadata descriptions in RELOAD make use of LOM standard terms which might not be readily understandable by many teachers. These factors have contributed to the design of a metadata facilitator which is intuitive and accessible for language teachers. A webform based metadata editing system is well suited in this case, in which certain LOM metadata fields are renamed with varied teacher-friendly terms along with optional hints for more detailed explanations so that it is more intuitive to use. In addition, the contextual metadata will be added or edited through the metadata facilitator as well, 
and this could be language teaching related information, such as "language specifics", "subject areas" and so on. Furthermore, the metadata facilitator will be integrated with the Wiki-type content editor so that both content and metadata can be modified from a single interface at the same time. A mechanism to allow users to create a standard content package from the repurposed materials and metadata has also been designed, which facilitates content packaging in the same system.

Since most practitioners are not experienced in using and attaching metadata, more features within the design have been provided to help them with the process. Firstly, a practitioner will be prompted to modify relevant metadata before a new version of learning object is saved; and he/she can get automated hints and suggestions while editing relevant metadata based on aspects of adaptation to the content. Meanwhile, the relationship between two revisions of the same learning object will be captured according to a user's selection. This relationship will be automatically recorded as a metadata field, for instance, a new revision is based on an earlier revision.

Table 1. Changes in metadata fields

\begin{tabular}{|c|c|c|}
\hline metadata & English Version & German Version \\
\hline Title & $\begin{array}{l}\text { Using the internet for } \\
\text { language learning }\end{array}$ & Im internet sprachen lernen \\
\hline Native language & British English & German/Deutsch \\
\hline Description & $\begin{array}{l}\text { This English language } \\
\text { resource reviews online } \\
\text { communication tools for } \\
\text { language learning and } \\
\text { includes links to different } \\
\text { web-based communication } \\
\text { tools for different languages }\end{array}$ & $\begin{array}{l}\text { This German language } \\
\text { resource reviews online } \\
\text { communication tools for } \\
\text { language learning, and includes } \\
\text { links to different web-based } \\
\text { communication tools for } \\
\text { different languages. }\end{array}$ \\
\hline keywords & $\begin{array}{l}\text { Internet, language, } \\
\text { learning, } \\
\text { communication, English }\end{array}$ & $\begin{array}{l}\text { Internet; language learning; } \\
\text { online communication; German }\end{array}$ \\
\hline Authors/creators & Watson, Julie & Watson, J. and Laxton, J. \\
\hline
\end{tabular}

\section{Discovery Agent}

With the aim of further supporting our community practitioners in terms of sharing and reusing learning objects, a tool to assist the discovery and selection of relevant objects is being developed. Practitioners can use this discovery agent interface to search across different repositories holding collections of learning objects. The search will be based on the metadata fields such as title, description and keywords. Specifically the wealth of pedagogical and contextual metadata attached to the learning objects can offer great benefits to practitioners who want to find certain types of objects according to their learning and teaching context. Moreover this tool will use an "online shopping trolley" metaphor for the selection and export of the RLOs. From a list of searching results, practitioners can select one or more relevant RLOs and add them to the learning object "trolley". The learning objects in the "trolley" can 
be viewed as practitioners wish and those RLOs can be removed from the "trolley" at any time. The RLOs can be checked out which means they are to be exported, possibly to the Wiki-type editor so that they can be repurposed.

\section{User Evaluation}

In order to check and validate whether the Wiki-type editing tool is appropriate and useful for teachers, we undertook a qualitative evaluation during a workshop given to language teachers and learning technologists from within the UK. The workshop once again highlighted the strong desire from practitioners to share online learning materials, whilst the lack of skills and the need for supporting tools to develop and repurpose learning objects.

$80 \%$ of the attendees were teachers who did not have much experience producing online learning materials or using a Wiki. They were asked to explore and use the tool to adapt a collection of existing learning objects. They were given a free choice as to the method they employed in order to evaluate the usability and effectiveness of the tool. Twenty one questionnaires were returned at the end of the session and during the session the development team observed the way the attendees interacted with the system. The general observation made was that most users managed to edit learning objects within a few minutes. They showed a great deal of enthusiasm towards the Wiki-type editor and were very keen to find out the possible modifications they could make to the learning objects. The observations provided valuable input for further improvements of the functionality of the editor.

The feedback we received from the questionnaires was positive. Some comments from the users are quoted here:

"...very easy to use and very effective..."

"... a good start, it could be more useful when more work has been done on it".

“...combines features of Dreamweaver, Word with Wiki tools. Nicely crafted tool with obvious utility”.

The analysis shows $86 \%$ of the users had a very good impression of the tool; $90 \%$ of them found it easy to navigate and obtain the information they wanted; $75 \%$ regarded it an effective tool for adapting learning objects; and 70\% considered it easy to become familiar with the tool. This indicated the editing tool is useful in repurposing learning objects and has the potential to be adopted in the current community. Many of the users can see how this tool could be used beyond its original scope. For example by including templates we could allow users to develop new and innovative learning objects from complicated components, something many find hard to do today.

\section{Conclusion and Ongoing Work}

Reuse and repurposing of learning objects has been much discussed but little practiced. Within the UK language teaching community there is a willingness and 
intent to reuse materials but the practitioners have identified their lack of technical expertise as a critical barrier. They have expressed their need for simple to use community tools. Furthermore, this trend can be observed throughout the World Wide Web where social and community approaches are embracing a whole new generation of computer users.

In this paper we have described our efforts to encourage and facilitate the reuse and repurposing of online learning objects within a Community of Practice by users with little technical expertise. The tools we created aim at this specific community to help discover, share and reuse HTML based learning objects. We have reasoned why a Wiki-type editing system could help such a community to adapt learning objects collaboratively via the Internet. We have explained the rationale for the design of an editing tool which reproduced the collaborative editing mechanisms of traditional Wikis for use by the community. The integration of a mature HTML editor has proved to be very effective in the case of adapting online learning materials for nonspecialist users. The tool has been evaluated and received positive reactions within a group of community members.

We identified from our earlier work that the addition of extended forms of contextual metadata attached to the learning materials would provide a wealth of new information with which to enhance the discovery and reusability of learning objects. But adding or modifying metadata could be very difficult for non-technical users without support. This leads to the metadata facilitator being developed which will bridge this gap. Finally we are building a discovery agent to facilitate resource discovery and selection. Ultimately all three tools will be integrated to form a single toolkit to help the management and reuse of learning objects.

\section{References}

1. Polsani, P.R.: Use and Abuse of Reusable Learning Objects. Journal of Digital Information 3(4) Article No. 164 (2003)

2. Barker, E., James, H., Knight, G.: Long-Term Retention and Reuse of E-Learning Objects and Materials. Report commissioned by the JISC (2004)

3. JISC funded project Sharing Language Learning Objects $(\mathrm{L}<$ Subscript $>2</$ Subscript $>0$ ). Available at, http://www.elanguages.ac.uk/sharing

4. Johnston, P.: It's all in the context. eFoundations metadata, middleware, e-learning (2006) Last accessed February 23, 2007, http://efoundations.typepad.com/efoundations/2006/12/ its_all_in_the_html

5. Specht, M., Kravcik, M.: Authoring of learning objects in context. International Journal on E-Learning 5, 25-33 (2006)

6. Jones, R.: Designing Adaptable Learning Resources with Learning Object Patterns. Journal of Digital Information 6(1) Article No. 305 (2004)

7. Jeffery, A.: Context, metadata and e-learning: a literature review. Project report (2006) Last accessed February 23, 2007 http://dash.ecs.soton.ac.uk/dokuwiki/doku.php? id=documents:context_metadata_and_e-learning_a_literature_review

8. Brooks, C., McCalla, G.: Towards flexible learning object metadata. International Journal of Continuing Engineering Education and Lifelong Learning 16(1/2) (2006) 
9. Pattuelli, M.C.: Context for content: Shaping learning objects and modeling a domain ontology. In: Blandford, A., Gow, J. (eds.) Digital Libraries in the Context of Users' Broader Activities. First International Workshop, DL-Cuba 2006, Chapel Hill, NC USA, DL-Cuba (2006)

10. Eduserv funded project MURLLO. Available at http://www.elanguages.ac.uk/murllo/

11. Wei, C., Maust, B., Barrick, J., Cuddihy, E., Spyridakis, J.H.: Wikis for Supporting Distributed Collaborative Writing. In: Wei, C., Maust, B., Barrick, J., Cuddihy, E., Spyridakis, J. (eds.) The Proceedings of the Society for Technical Communication 52nd Annual Conference, Seattle, WA, May 8-11 (2005)

12. Kravcik, M., Specht, M.: Authoring Adaptive Courses - ALE Approach. Advanced Technology for Learning 1(4), 215-220 (2004)

13. Leuf, B., Cunningham, W.: The Wiki way, quick collaboration on the Web. AddisonWesley, Boston (2001) 